\title{
Impact of ejaculatory abstinence period and semen characteristic on the reproductive outcomes after intracytoplasmic sperm injection
}

\author{
Elham Azizi ${ }^{1}$, Mohammad Naji ${ }^{2}$, Saghar Salehpour ${ }^{3,4}$, Nasrin Saharkhiz ${ }^{3,4}$, Maryam Karimi ${ }^{3}$, Nasrin Borumandnia ${ }^{2}$, \\ Zahra Shams Mofarahe ${ }^{1,5}$ \\ ${ }^{1}$ Department of Biology and Anatomical Sciences, School of Medicine, Shahid Beheshti University of Medical \\ Sciences, Tehran, Iran \\ ${ }^{2}$ Urology and Nephrology Research Center (UNRC), Shahid Beheshti University of Medical Sciences, Tehran, Iran \\ ${ }^{3}$ IVF center, Taleghani Hospital, Shahid Beheshti University of Medical Sciences, Tehran, Iran \\ ${ }^{4}$ Preventative Gynecology Research Center (PGRC), Imam Hossein Hospital, Shahid Beheshti University of \\ Medical Sciences, Tehran, Iran \\ ${ }^{5}$ Taleghani Hospital Research Development Unit, School of Medicine, Shahid Beheshti University of Medical \\ Sciences, Tehran, Iran
}

\begin{abstract}
Objective: The prognostic of semen characteristics in intracytoplasmic sperm injection (ICSI) outcomes is not clear. Also, there is no evidence-based recommendation for the abstinence period before ICSI. So, we aimed to assess the influence of the abstinence period and semen characteristics on ICSI outcomes.

Methods: A total of 1003 fresh ICSI cycles were divided into six groups; group 1 (1-day abstinence), group 2 ( 2 days abstinence), group 3 ( 3 days abstinence), group 4 (4 days abstinence), group 5 (5 days abstinence), and group 6 (6-10 days abstinence).

Results: We showed that semen volume $(p=0.0001)$ and total sperm count $(p=0.005)$ were increased in the groups with higher abstinence periods. Other semen parameters did not significantly associate with the abstinence period. The percentage of progressively motile sperm was associated with fertilization rate $(p=0.007)$, and the sperm morphology was associated with cleavage-stage embryo rate $(p=0.036)$. No influence of abstinence or semen parameters on rates of pregnancies was observed.

Conclusions: The abstinence period before ICSI can influence the semen volume and total sperm count, and possibly fertilization. Although the sperm with the highest quality are selected for ICSI, the percentages of progressively motile and morphologically normal sperm in the ejaculated semen have a predictive value for fertilization and cleavage rates after ICSI, respectively.
\end{abstract}

Keywords: ICSI, fertilization, pregnancy, sexual abstinence, semen

\section{INTRODUCTION}

Spermatogenesis takes place in the seminiferous tubules of the testis, and then the immature spermatozoa migrate to the epididymis, where they develop their final state of maturity in the male reproductive tract and are stored in cauda epididymis until ejaculation (Rowley et al., 1970; Amann \& Howards, 1980; Johnson, 1982; Johnson \& Varner, 1988; Robaire et al., 2006). The sexual abstinence period, duration of the mature sperm storage in the epididymis can influence semen parameters. Longer sexual abstinence leads to increased semen volume and sperm count (Raziel et al., 2001; De Jonge et al., 2004; Jurema et al., 2005; Sobreiro et al., 2005; Marshburn et al., 2010; Sunanda et al., 2014; Agarwal et al., 2016; Welliver et al., 2016; Hanson et al., 2018). However, the influence of the abstinence period on other semen quality parameters such as sperm motility, morphology, viability, and DNA integrity is still inconclusive and controversial (Hanson et al., 2018). Although the abstinence period may affect some semen parameters, the optimal abstinence period for assisted reproductive technology (ART) cycles has remained to be elucidated.

Currently, most ART clinics use the World Health Organization (WHO) recommendation for a sexual abstinence period (2-7 days) before ART cycles. WHO recommended this period for the evaluation of semen quality in the primary assessment of male fertility (WHO, 2010). In this regard, the European Society of Human Reproduction and Embryology (ESHRE) has suggested a shorter period of sexual abstinence (3-4 days) for semen quality evaluation (Barratt et al., 2011). Nevertheless, the WHO recommendation on the sexual abstinence period has been challenged by some studies that reported superior semen quality in different abstinence days from the recommended period (De Jonge et al., 2004; Levitas et al., 2005; Mayorga-Torres et al., 2015; Agarwal et al., 2016).

The optimal abstinence period may improve some functional semen parameters, with substantial roles in fertilization potential, which cannot be appraised in routine semen quality evaluation. Hence, it is necessary to determine the optimal abstinence period in which human spermatozoa have the highest fertilization potential for improvement of ART success rate. Concerning the optimal sexual abstinence period for ART procedures, there are a few studies with contradictory findings. Some studies have reported that a shorter abstinence period can improve pregnancy outcomes in intracytoplasmic sperm injection (ICSI) cycles (Colturato et al., 2007; Periyasamy et al., 2017; Borges Jr et al., 2019), but another group has reported no correlation between abstinence period and ICSI outcomes (Lee et al., 2015; 2018). Lee et al. (2018) have even indicated that the abstinence period beyond the recommended period of WHO did not decline pregnancy outcomes. Furthermore, semen samples collected after recurrent ejaculations and final abstinence of 12 hours could improve pregnancy outcomes in ICSI cycles (Sánchez-Martín et al., 2013). The highest pregnancy outcomes with IUI were observed following less than three days of abstinence (Sugiyam et al., 2008)2008. These findings have questioned the clinical application of WHO and ESHRE recommendations for the duration of the sexual abstinence period in ART (Ayad et al., 2018). Therefore, defining the optimal sexual abstinence period for clinical application in ART should be considered by WHO and ESHRE. 
ICSI procedure is the most widely used technique in ART, which may utilize for male or non-male factor infertility (European IVF-monitoring Consortium, 2017). Although in this procedure, only one sperm is selected by the embryologist for the injection into the cytoplasm of the oocyte, the semen quality may have prognostic value for the success of the ICSI. However, the literature regarding the prognostic value of the semen parameters, which may be affected by the abstinence period, on the ICSI outcomes are scanty, and the association is still under debate. However, some recent studies have been reported the semen quality might influence the outcomes of ICSI cycles (Chapuis et al., 2017; Mazzilli et al., 2017; Bartolacci et al., 2018).

The influence of the abstinence period and also semen quality on the subsequent ICSI cycle outcomes is a conflicting issue in the literature (Borges Jr et al., 2019; Setti et al., 2020). Therefore, in this study, we evaluated the association between the sexual abstinence period and semen quality, fertilization rate, cleavage-stage embryo rate, and pregnancy outcomes after fresh ICSI cycles.

\section{MATERIAL AND METHODS}

This retrospective study has assessed 1003 patients who underwent ICSI cycles, from December 2014 to August 2019. Fresh embryo transferred (ET) ICSI cycles were only included in this study. ICSI cycles that used sperm from testicular biopsies, males with hepatitis, pre-implantation genetic diagnosis, donation or activation of oocytes, frozen sperm, and oocyte in vitro maturation (IVM) were excluded. All procedures were performed according to guidelines of the ethics committee and informed consent was waived for the current study (IR.SBMU.RETECH. REC.1398.201).

\section{Semen collection and sperm preparation}

The sexual abstinence period was asked before collecting semen samples. Semen samples were collected in our laboratory at the time of oocyte retrieval by masturbation into a wide-mouth specimen container. Samples were liquefied at $37^{\circ} \mathrm{C}$ for $30-60 \mathrm{~min}$, before evaluation. Analysis of neat semen was performed for volume, sperm count, motility, and morphology according to the WHO criteria (WHO, 2010). Sperm concentration was the number of spermatozoa/mL in the semen. The total sperm count was determined by multiplying the sperm concentration by the volume of ejaculated semen. The percentage of motile sperm was evaluated among 200 spermatozoa in the semen, and the total sperm motility was the sum of the number of progressive and non-progressive spermatozoa. The percentage of the morphologically normal sperm was assessed using the Diff-quick method (Diff-Quick, Quick-Panoptic, Amposta, Spain). The swim-up method was used for the preparation of sperm before the ICSI procedure.

\section{Controlled ovarian stimulation, oocyte retrieval, and ICSI}

Ovarian stimulation was performed either with $\mathrm{GnRH}$ antagonist or agonist protocols, based on specific parameters of the patients such as female age, body mass index (BMI), ovarian reserve, and medical history. Stimulation in patients was initiated with recombinant FSH (Gonal-F, Merck Serono Europe Ltd, UK). When at least 3 follicles $>17$ $\mathrm{mm}$ diameter were visualized by ultrasonography, human chorionic gonadotropin (hCG) (Pregnyl; MSD, Brussels, Belgium) trigger was administrated. 34-38 hours after hCG administration, cumulus-oocyte complexes were retrieved from both ovaries, by transvaginal aspiration under ultrasound guidance. After denudation of retrieved oocytes, and removal of surrounding cumulus cells, all matured oocytes were inseminated.
Embryo transfer and luteal phase support

The embryos were cultured in a Global medium (Life Global ${ }^{\circledR}$ ART media) and incubated in $6 \% \mathrm{CO}_{2}$ at $37^{\circ} \mathrm{C}$. Between 18-20 hours after insemination, fertilization was monitored by the presence of two pronuclei and two clear polar bodies in perivitelline space. Embryos were assessed and graded in cleavage-stage according to the number, size, and symmetry of blastomeres and the degree of fragmentation (Puissant et al., 1987). For embryo transfer, 1-3 embryos were transferred to the uterine either on day 2 or 3. Biochemical pregnancy was determined by measuring beta-hCG levels in the blood sample test on day 14 after oocyte retrieval. Clinical pregnancy was determined by visualization of a gestational sac in ultrasound at weeks 5-6.

\section{Data analysis}

Based on the sexual abstinence period, the cycles were divided into six groups $(1,2,3,4,5$, and 6-10 days of abstinence). The main outcome measures of this study were the rates of fertilization, cleavage-stage embryos, biochemical and clinical pregnancies.

Statistical analysis was carried out using SPSS software 22 (SPSS Inc., Chicago, IL). Data were presented as mean \pm standard deviation for continuous variables and frequency (percentage) for categorical variables. Kruskal-Wallis (with Dunn's multiple comparisons test) and $X^{2}$ test were utilized for analysis of continuous and categorical variables, respectively.

In the multivariable analysis, we assessed the effect of abstinence period and also semen parameters (semen volume, total sperm count, sperm concentration, and the percentage of total motile, progressively motile, and morphologically normal sperm) on the main outcome measures. Female age, male age, early or secondary fertility, previous cycles, stimulation, duration of infertility, number of aspirated oocytes, number of transferred embryos, etiology (combined vs. Other, male factor $v s$. Other, unexplained vs. Other), ovarian stimulation protocol (antagonist vs. agonist) were also considered as potential confounding variables. To adjust the effects of abstinence period, semen parameters, and other confounding variables, multivariate logistic regression, and multiple linear regression were performed for dichotomous (biochemical pregnancy and clinical pregnancy), and continuous outcomes (fertilization and cleavage-stage embryo rate), respectively. A $p$-value of $<0.05$ was considered statistically significant.

\section{RESULTS}

A total of 1003 fresh ICSI cycles were analyzed in this study and categorized into six groups based on days of abstinence period; group 1 ( 1 day, $n=37)$, group 2 (2 days, $\mathrm{n}=170$ ), group 3 ( 3 days, $\mathrm{n}=392)$, group 4 (4 days, $n=253$ ), group 5 ( 5 days, $n=102)$, and group 6 (6-10 days, $\mathrm{n}=49$ ). From 1003 studied patients, 21 patients had more than 7 days of abstinence period, which were more than WHO recommendation. No significant differences were detected in baseline characteristics of subjects in different studied groups, except for female age differences between groups 2 vs. $4(p=0.01)$ (Table 1$)$.

Among ICSI cycle variables, there were no significant differences in the number of aspirated, injected, and fertilized oocytes, and the number of transferred embryos. In studied semen parameters, semen volume and also total sperm count were significantly increased in the groups with higher abstinence periods $(p=0.0001$ and $p=0.005$, respectively). Sperm concentration, the percentage of total motile, progressively motile, and morphologically normal sperm did not significantly differ among studied groups (Table 2 ). The highest rate of biochemical (32.4\%) and clinical (31.1\%) pregnancy were noticed in group 5 ( 5 days of abstinence) but no significant differences were 
Table 1. Demographic and cycle characteristics of patients undergoing ICSI.

\begin{tabular}{|c|c|c|c|c|c|c|c|}
\hline \multirow[b]{2}{*}{ Parameter } & \multicolumn{6}{|c|}{ Abstinence period } & \multirow[b]{2}{*}{$p$-value } \\
\hline & $\begin{array}{c}\text { Group 1 } 1^{a} \\
(n=37)\end{array}$ & $\begin{array}{l}\text { Group 2a } \\
(n=170)\end{array}$ & $\begin{array}{l}\text { Group } 3^{a} \\
(n=392)\end{array}$ & $\begin{array}{l}\text { Group 4a } \\
(n=253)\end{array}$ & $\begin{array}{l}\text { Group } 5^{a} \\
(n=102)\end{array}$ & $\begin{array}{c}\text { Group 6a } \\
(n=49)\end{array}$ & \\
\hline Female age (y) & $33 \pm 6.1$ & $34 \pm 5.9^{b}$ & $33 \pm 6.0$ & $32 \pm 5.9^{b}$ & $34 \pm 5.6$ & $32 \pm 5.1$ & 0.01 \\
\hline Male age $(y)$ & $37 \pm 5.1$ & $38 \pm 6.3$ & $37 \pm 6.4$ & $36 \pm 5.4$ & $37 \pm 6.7$ & $36 \pm 5.9$ & 0.1 \\
\hline $\begin{array}{l}\text { Type of infertility } \\
\text { Primary } \mathrm{n}(\%) \\
\text { Secondary } \mathrm{n}(\%)\end{array}$ & $\begin{array}{c}30(81.1) \\
7(18.9)\end{array}$ & $\begin{array}{c}131(77.5) \\
38(22.5)\end{array}$ & $\begin{array}{c}328(83.7) \\
64(16.3)\end{array}$ & $\begin{array}{l}201(79.5) \\
52(20.5)\end{array}$ & $\begin{array}{l}81(79.4) \\
21(20.6)\end{array}$ & $\begin{array}{c}44(89.8) \\
5(10.2)\end{array}$ & 0.2 \\
\hline Infertility duration $(y)$ & $6.7 \pm 7.1$ & $6.2 \pm 5.6$ & $5.4 \pm 4.2$ & $4.7 \pm 3.7$ & $5.3 \pm 4.2$ & $4.5 \pm 4.0$ & 0.1 \\
\hline $\begin{array}{l}\text { No. of previous ICSI cydes } \mathbf{N}(\%) \\
0 \\
1 \\
2 \\
3 \text { \& more }\end{array}$ & $\begin{array}{c}27(72.9) \\
5(13.5) \\
3(8.1) \\
2(5.4) \\
\end{array}$ & $\begin{array}{c}114(68.7) \\
31(18.7) \\
17(10.2) \\
4(2.4)\end{array}$ & $\begin{array}{c}269(68.6) \\
74(18.8) \\
33(8.5) \\
16(4.1)\end{array}$ & $\begin{array}{c}170(67.2) \\
56(22.1) \\
21(8.3) \\
6(2.4)\end{array}$ & $\begin{array}{c}70(68.6) \\
22(21.6) \\
6(5.9) \\
4(3.9) \\
\end{array}$ & $\begin{array}{c}36(73.5) \\
9(18.4) \\
3(6.1) \\
1(2) \\
\end{array}$ & 0.9 \\
\hline $\begin{array}{l}\text { Etiology } \mathbf{N}(\%) \\
\text { Male infertility } \\
\text { PCOS } \\
\text { Endometriosis } \\
\text { Tubal factor } \\
\text { Uterine } \\
\text { Endocrinologydisorder } \\
\text { Unexplained } \\
\text { Combined }\end{array}$ & $\begin{array}{c}7(18.9) \\
1(2.8) \\
0(0) \\
0(0) \\
0(0) \\
0(0) \\
3(8.1) \\
26(70.2)\end{array}$ & $\begin{array}{c}20(11.8) \\
6(3.5) \\
0(0) \\
2(1.2) \\
8(4.7) \\
7(4.1) \\
9(5.3) \\
118(69.4)\end{array}$ & $\begin{array}{c}82(20.9) \\
18(4.6) \\
1(0.3) \\
16(4.1) \\
3(0.7) \\
15(3.8) \\
24(6.1) \\
234(59.5)\end{array}$ & $\begin{array}{c}46(18.2) \\
10(4) \\
0(0) \\
6(2.4) \\
1(0.4) \\
5(2) \\
7(2.7) \\
178(70.3)\end{array}$ & $\begin{array}{c}26(25.5) \\
5(4.9) \\
0(0) \\
5(4.9) \\
2(2) \\
3(2.9) \\
4(3.9) \\
57(55.9)\end{array}$ & $\begin{array}{c}5(10.2) \\
1(2.1) \\
0(0) \\
1(2) \\
1(2) \\
1(2) \\
3(6.1) \\
37(75.6)\end{array}$ & 0.06 \\
\hline $\begin{array}{l}\text { Stimulation protocol } \\
\text { Antagonist N (\%) } \\
\text { Agonist N (\%) }\end{array}$ & $\begin{array}{c}37(100) \\
0(0)\end{array}$ & $\begin{array}{c}161(94.7) \\
9(5.3)\end{array}$ & $\begin{array}{c}361(92.1) \\
31(7.9)\end{array}$ & $\begin{array}{c}238(94.07) \\
15(5.92)\end{array}$ & $\begin{array}{c}238(94.07) \\
15(5.92)\end{array}$ & $\begin{array}{c}47(95.9) \\
2(4.1)\end{array}$ & 0.2 \\
\hline
\end{tabular}

Data were presented as mean \pm SD or frequency (percentage).

a The ICSI cycles were divided into six groups based on days of abstinence period: Group 1: 1 day abstinence, Group 2: 2 days abstinence, Group 3: 3 days abstinence, Group 4: 4 days abstinence, Group 5: 5 days abstinence, Group 6: 6-10 days abstinence

b Values with the same superscript in a row were significantly different.

Table 2. Characteristics and outcomes of ICSI cycles.

\begin{tabular}{|c|c|c|c|c|c|c|c|}
\hline \multirow[b]{2}{*}{ Parameter } & \multicolumn{6}{|c|}{ Abstinence period } & \multirow[b]{2}{*}{$p$-value } \\
\hline & $\begin{array}{c}\text { Group 1 } \\
(n=37)\end{array}$ & $\begin{array}{l}\text { Group } 2^{a} \\
(n=170)\end{array}$ & $\begin{array}{l}\text { Group } 3^{a} \\
(n=392)\end{array}$ & $\begin{array}{l}\text { Group 4a } \\
(n=253)\end{array}$ & $\begin{array}{l}\text { Group } 5^{a} \\
(n=102)\end{array}$ & $\begin{array}{l}\text { Group } 6^{a} \\
(n=49)\end{array}$ & \\
\hline Semen volume $(\mathrm{mL})$ & $2.6 \pm 1.4^{c}$ & $2.9 \pm 1.5^{b}$ & $3.4 \pm 1.5^{\mathrm{cb}}$ & $3.4 \pm 1.5 c^{b}$ & $3.6 \pm 1.5^{\mathrm{cb}}$ & $3.6 \pm 1.7^{c}$ & $<0.0001$ \\
\hline Total sperm count & $118 \pm 126^{c}$ & $139 \pm 117^{b}$ & $160 \pm 134$ & $157 \pm 115$ & $196 \pm 127$ & $213 \pm 158^{\mathrm{cb}}$ & 0.005 \\
\hline Sperm concentration & $46 \pm 41$ & $52 \pm 37$ & $49 \pm 30$ & $50 \pm 32$ & $49 \pm 30$ & $61 \pm 36$ & 0.1 \\
\hline Total sperm motility (\%) & $51 \pm 18$ & $51 \pm 20$ & $54 \pm 19$ & $51 \pm 20$ & $48 \pm 20$ & $54 \pm 19$ & 0.05 \\
\hline Progressive motility of sperm (\%) & $41 \pm 21$ & $41 \pm 19$ & $43 \pm 19$ & $42 \pm 19$ & $40 \pm 20$ & $44 \pm 20$ & 0.6 \\
\hline Sperm morphology (\%) & $1.8 \pm 2.3$ & $2.1 \pm 2.0$ & $2.3 \pm 2.0$ & $2.2 \pm 1.9$ & $2.6 \pm 2.2$ & $2.2 \pm 2.0$ & 0.2 \\
\hline No. oocytes aspirated & $8.8 \pm 6.5$ & $8.2 \pm 6.2$ & $8.4 \pm 5.9$ & $7.8 \pm 6.1$ & $8.3 \pm 5.8$ & $8.6 \pm 6.3$ & 0.5 \\
\hline No. oocytes injected & $6.8 \pm 5.3$ & $6.4 \pm 4.8$ & $6.6 \pm 4.9$ & $6.3 \pm 5.1$ & $6.4 \pm 4.1$ & $6.9 \pm 5.4$ & 0.8 \\
\hline No. oocytes fertilized & $4.4 \pm 3.7$ & $4.6 \pm 3.8$ & $4.7 \pm 3.7$ & $4.3 \pm 4.1$ & $4.6 \pm 3.3$ & $5.0 \pm 4.6$ & 0.4 \\
\hline No. embryos transferred & $1.6 \pm 0.5$ & $1.9 \pm 0.4$ & $1.8 \pm 0.5$ & $1.9 \pm 0.4$ & $1.9 \pm 0.5$ & $1.7 \pm 0.5$ & 0.03 \\
\hline Fertilization rate (\%) & $66 \pm 32$ & $72 \pm 30$ & $73 \pm 27$ & $68 \pm 32$ & $67 \pm 29$ & $69 \pm 32$ & 0.4 \\
\hline Cleavage-stage embryo rate (\%) & $89 \pm 29$ & $91 \pm 25$ & $92 \pm 22$ & $91 \pm 23$ & $92 \pm 24$ & $93 \pm 16$ & 0.8 \\
\hline $\begin{array}{l}\text { Biochemical pregnancy rate per } \\
\text { embryo transfer } N(\%)\end{array}$ & $8(29.1)$ & $35(29.4)$ & $90(30.2)$ & $58(30.0)$ & $24(32.4)$ & $11(27.8)$ & 0.9 \\
\hline $\begin{array}{l}\text { Clinical pregnancy rate per } \\
\text { embryo transfer } N(\%)\end{array}$ & $8(29.2)$ & $30(25.2)$ & $84(28.1)$ & $52(26.9)$ & $23(31.1)$ & $11(27.8)$ & 0.9 \\
\hline
\end{tabular}

Data were presented as mean \pm SD or frequency (percentage).

a The ICSI cycles were divided into six groups based on days of abstinence period: Group 1: 1 day abstinence, Group 2: 2 days abstinence, Group 3: 3 days abstinence, Group 4: 4 days abstinence, Group 5: 5 days abstinence, Group 6: 6-10 days abstinence

$b, c$ Values with the same superscript in a row were significantly different. 
observed among studied groups. There were also no significant differences in fertilization and cleavage-stage embryo rates of the studied groups (Table 2 ).

A multivariable analysis was carried out to adjust the outcome measures of the study for potentially confounding variables (Table 3 ). It was revealed that the progressive motility of sperm was associated with fertilization rate $(\mathrm{B}=1.48, \mathrm{CI}=0.40,2.55, p=0.007)$ (Table 3). Moreover, sperm morphology was associated with cleavage-stage embryo rate $(\mathrm{B}=-0.64, \mathrm{CI}=-1.23,-0.041, p=0.036)$ (Table 3 ). There is not a significant association between the abstinence period and the fertilization rate $(B=-1.42, C I=$ $-3.05,0.20, p=0.087$ ) (Table 3 ). Nevertheless, there was no association between abstinence or semen parameters and rates of pregnancies.

\section{DISCUSSION}

To date, there is no clear, evidence-based recommendation for the sexual abstinence period before the ICSI treatment of infertile couples. We analyzed the outcome of 1003 fresh ICSI cycles to assess the importance of the sexual abstinence period and semen quality in the cycles. However, our findings demonstrated that some ICSI outcomes were associated with specific sperm parameters. The sexual abstinence period could affect some characteristics of the ejaculated semen.

It was reported in a systematic review that the effects of sexual abstinence on sperm parameters have shown that longer abstinence was associated with increased semen volume and total sperm count (Hanson et al., 2018), but reports regarding the impact of abstinence on other semen parameters were contradictory and inconclusive
(Hanson et al., 2018). According to our findings, longer sexual abstinence results in higher total sperm count and increased semen volume, which is in accordance with previous reports (Raziel et al., 2001; De Jonge et al., 2004; Jurema et al., 2005; Sobreiro et al., 2005; Marshburn et al., 2010; Sunanda et al., 2014; Agarwal et al., 2016; Welliver et al., 2016; Hanson et al., 2018). Besides, we noticed that the concentration, motility, and morphology of the sperm in the ejaculated semen were not affected by the duration of the sexual abstinence between 1 to 10 days. The longer sexual abstinence period resulted in the accumulation of the sperm in the epididymis, so, there was a high number of sperm in the ejaculated semen and also the semen volume was increased.

In this study, biochemical and clinical pregnancy and also cleavage-stage embryo rates were not significantly associated with sexual abstinence. Nevertheless, we found no significant association between sexual abstinence periods before ICSI and fertilization rate after the procedure. There are several studies with the same results in this regard (Colturato et al., 2007; Lee et al., 2015; 2018; Periyasamy et al., 2017; Borges Jr et al., 2019; Setti et al., 2020). Lee et al. (2015) in a retrospective study have evaluated fertilization and pregnancy outcomes of 131 fresh ICSI cycles regarding different abstinence periods (2-4 days vs. 5-7 days). They found no significant difference between two groups of abstinence periods in fertilization rate, top-quality embryos on day 3, and clinical pregnancy rate (Lee et al., 2015). Another retrospective analysis of 449 fresh ICSI cycles has compared the clinical outcomes between different abstinence periods (2-7 days vs. $\geq 8$ days) in groups of young maternal age ( $<38$ years) and old maternal age ( $\geq 38$ years). They demonstrated

\begin{tabular}{|c|c|c|c|}
\hline Multivariable linear regression analysis of Fertilization rate ${ }^{a b}$ & Beta & CI for Beta & p-value \\
\hline Abstinence period (days) & -1.42 & $-3.05,0.20$ & 0.087 \\
\hline Duration of infertility $(Y)$ & -0.70 & $-1.20,-0.21$ & 0.005 \\
\hline Progressive motility of sperm & 1.48 & $0.40,2.55$ & 0.007 \\
\hline No. top-quality embryos transferred & 6.33 & $1.71,10.95$ & 0.007 \\
\hline No. retrieved oocytes & -1.17 & $-1.75,-0.60$ & $<0.001$ \\
\hline Multivariable linear regression analysis of Cleavage-stage embryo rate ${ }^{\text {ab }}$ & Beta & CI for Beta & p-value \\
\hline Early or secondary infertility & -3.52 & $-6.27,-0.77$ & 0.012 \\
\hline Progressive motility of sperm & 0.06 & $-0.002,0.128$ & 0.056 \\
\hline Sperm morphology & -0.64 & $-1.23,-0.041$ & 0.036 \\
\hline No. oocytes aspirated & -0.35 & $-0.68,-0.06$ & 0.017 \\
\hline Multivariable logistic regression analysis of Implantation ab & OR & CI for OR & p-value \\
\hline Female age $(Y)$ & 0.96 & $0.92,1.00$ & 0.089 \\
\hline Etiology (combined vs. all) & 2.05 & $1.30,3.22$ & 0.002 \\
\hline \multicolumn{4}{|l|}{ No. top-quality embryos transferred } \\
\hline & 1.92 & $1.20,3.09$ & 0.007 \\
\hline Multivariable logistic regression analysis of Clinical pregnancy rate ${ }^{\text {ab }}$ & OR & CI for OR & p-value \\
\hline Female age $(\mathrm{Y})$ & 0.96 & $0.92,1.00$ & 0.072 \\
\hline Etiology (combined vs. all) & 1.98 & $1.25,3.14$ & 0.003 \\
\hline
\end{tabular}

a: Regression modeling with a Backward approach.

b: Variables entered for multivariable regression analysis: abstinence period, female age, male age, early or secondary infertility, previous ICSI cycles, ovarian stimulation protocol (agonist vs. antagonist), duration of infertility, semen volume, total sperm count, motility, progressive motility, sperm concentration, sperm morphology, number of aspirated oocytes, number of transferred embryos, etiology (combined $v s$. other, male factor $v s$. Other, unexplained $v s$. other). CI: confidence interval. 
that fertilization rate, top-quality embryos on day 3, and pregnancy rate were not significantly different between 2-7 and $\geq 8$ days of abstinence. They have also suggested that the sexual abstinence beyond the recommended abstinence period by WHO (2-7 days) was not associated with lower fertilization and pregnancy rates in ICSI cycles (Lee et al., 2018).

However, several of the preceding studies have indicated the sexual abstinence period was associated with embryo development and pregnancy outcomes after the ICSI procedure (Colturato et al., 2007; Periyasamy et al., 2017; Borges Jr et al., 2019; Setti et al., 2020). Setti et al. (2020) in a cohort study involving 427 oocyte recipient ICSI cycles showed that the abstinence period could affect embryo development and implantation. Moreover, in a prospective cohort study by Borges Jr et al. (2019), data from 818 ICSI patients were analyzed and the results showed that the ejaculatory abstinence period could influence implantation and pregnancy rates. They showed that 1-day of abstinence led to higher implantation and pregnancy rates compared to 2-4 days of abstinence (Borges Jr et al., 2019). Colturato and colleagues, in a retrospective study of 445 ICSI cycles, compared pregnancy outcomes of ICSI cycles in different sexual abstinence periods $(1,2,3,4,5$, $6-10$, and $\geq 11$ days of abstinence). They showed that the highest pregnancy rate was achieved in 1-day abstinence before ICSI, and the lowest pregnancy rate was found in 5 days abstinence period. Also, five or more days of sexual abstinence period were associated with a decreased pregnancy rate. Also, they reported that a shorter sexual abstinence period was associated with higher embryo quality and an increased pregnancy rate (Colturato et al., 2007). Periyasamy et al. (2017) analyzed outcomes of 1030 ICSI/ IVF cycles according to the duration of the sexual abstinence period in two groups (2-7 days vs. $>7$ days of abstinence). They demonstrated that significantly higher implantation, pregnancy, and live birth rates were achieved in patients with 2-7 days of abstinence period, in comparison to $>7$ days of abstinence. Moreover, the highest pregnancy and live birth rates were noticed in a subgroup of patients with 2-4 days of abstinence period.

The results of our study showed that progressive motility of sperm could affect the fertilization rate in the ICSI cycle. Although there is no natural sperm selection in ICSI procedure and the best spermatozoa in the semen sample could be selected, Bartolacci et al. (2018) have reported that sperm motility was associated with fertilization rate but not with implantation in ICSI cycles. In accordance with our findings, they also have reported that the pregnancy outcomes of ICSI cycles did not associate with semen parameters (Bartolacci et al., 2018). It has been proposed that disrupted spermatogenesis and DNA damages which lead to the development of an abnormal flagellum may affect the motility of spermatozoa (Waller et al., 2019). Because of this, the significant positive association between progressive sperm motility and fertilization rate in the ICSI cycle might be due to possible disruption during spermatogenesis. There is very little evidence regarding the role of sperm morphology on embryo development (Danis \& Samplaski, 2019), even so, our data demonstrated that the percentage of morphologically normal sperm was negatively associated with cleavage-stage embryo rate. This finding may indicate the importance role of sperm morphological abnormalities on the early stages of the embryo development.

The sexual abstinence period between 1 to 10 days has a significant association with the total sperm count and semen volume. The fertilization rate in the ICSI cycle is also associated with the progressive sperm motility in the preprocessing semen. The cleavage-stage embryo rate is negatively associated with the percentage of morphologically normal sperm in the preprocessing semen. Further, the pregnancy outcomes of the ICSI cycles are not affected by the abstinence period or the semen characteristics. We recommend future studies in a larger population to prove the impact of the abstinence period on the outcomes of ICSI cycles and also the prognostic value of semen parameters in the cycles.

\section{ACKNOWLEDGMENT}

The authors thank the personnel of Taleghani IVF center for valuable assistance. The study was supported by the Taleghani Hospital Research Development Unit, Shahid Beheshti University of Medical Sciences (IR.SBMU.RETECH.REC.1398.201).

\section{CONFLICT OF INTEREST}

None.

\section{Corresponding author:}

Zahra Shams Mofarahe

Department of Biology and Anatomical Sciences

School of Medicine,

Shahid Beheshti University of Medical Sciences

Tehran, Iran.

E-mail: z_shams@sbmu.ac.ir

\section{REFERENCES}

Agarwal A, Gupta S, Du Plessis S, Sharma R, Esteves SC, Cirenza C, Eliwa J, Al-Najjar W, Kumaresan D, Haroun N, Philby S, Sabanegh E. Abstinence Time and Its Impact on Basic and Advanced Semen Parameters. Urology. 2016;94:102-10. PMID: 27196032 DOI: 10.1016/j.urology.2016.03.059

Amann RP, Howards SS. Daily spermatozoal production and epididymal spermatozoal reserves of the human male. J Urol. 1980;124:211-5. PMID: 6772801 DOI: 10.1016/ S0022-5347(17)55377-X

Ayad BM, Horst GV, Plessis SSD. Revisiting The Relationship between The Ejaculatory Abstinence Period and Semen Characteristics. Int J Fertil Steril. 2018;11:238-46. PMID: 29043697 DOI: $10.22074 /$ ijfs.2018.5192

Barratt CL, Björndahl L, Menkveld R, Mortimer D. ESHRE special interest group for andrology basic semen analysis course: a continued focus on accuracy, quality, efficiency and clinical relevance. Hum Reprod. 2011;26:3207-12. PMID: 21965314 DOI: 10.1093/humrep/der312

Bartolacci A, Pagliardini L, Makieva S, Salonia A, Papaleo $\mathrm{E}$, Viganò P. Abnormal sperm concentration and motility as well as advanced paternal age compromise early embryonic development but not pregnancy outcomes: a retrospective study of 1266 ICSI cycles. J Assist Reprod Genet. 2018;35:1897-903. PMID: 29995229 DOI: 10.1007/ s10815-018-1256-8

Borges E Jr, Braga DPAF, Zanetti BF, Iaconelli A Jr, Setti AS. Revisiting the impact of ejaculatory abstinence on semen quality and intracytoplasmic sperm injection outcomes. Andrology. 2019;7:213-9. PMID: 30570220 DOI: 10.1111/andr.12572

Chapuis A, Gala A, Ferrières-Hoa A, Mullet T, Bringer-Deutsch S, Vintejoux E, Torre A, Hamamah S. Sperm quality and paternal age: effect on blastocyst formation and pregnancy rates. Basic Clin Androl. 2017;27:2. PMID: 28127436 DOI: $10.1186 /$ s12610-016-0045-4 
Colturato S, Abdelmassih S, Carizza C, Nagy P, Abdelmassih $\mathrm{V}$, Abdelmassih $\mathrm{R}$. Influence of sexual abstinence length on sperm parameters and on IVF outcomes in ICSI assisted treatment cycles. Fertil Steril. 2007;88:S252. DOI: $10.1016 /$ j.fertnstert.2007.07.861

Danis RB, Samplaski MK. Sperm Morphology: History, Challenges, and Impact on Natural and Assisted Fertility. Curr Urol Rep. 2019;20:43. PMID: 31203470 DOI: $10.1007 / \mathrm{s} 11934-019-0911-7$

De Jonge $C$, LaFromboise $M$, Bosmans $E$, Ombelet $W$, Cox A, Nijs $M$. Influence of the abstinence period on human sperm quality. Fertil Steril. 2004;82:57-65. PMID: 15236990 DOI: $10.1016 /$ j.fertnstert.2004.03.014

European IVF-monitoring Consortium (EIM); European Society of Human Reproduction and Embryology (ESHRE), Calhaz-Jorge C, De Geyter C, Kupka MS, de Mouzon J, Erb K, Mocanu E, Motrenko T, Scaravelli G, Wyns C, Goossens V. Assisted reproductive technology in Europe, 2013: results generated from European registers by ESHRE. Hum Reprod. 2017;32:1957-73. PMID: 29117383 DOI: 10.1093/ humrep/dex264

Hanson BM, Aston KI, Jenkins TG, Carrell DT, Hotaling JM. The impact of ejaculatory abstinence on semen analysis parameters: a systematic review. J Assist Reprod Genet. 2018;35:213-20. PMID: 29143943 DOI: 10.1007/s10815017-1086-0

Johnson L. A re-evaluation of daily sperm output of men. Fertil Steril. 1982;37:811-6. PMID: 7084502 DOI: $10.1016 /$ S0015-0282(16)46343-0

Johnson L, Varner DD. Effect of daily spermatozoan production but not age on transit time of spermatozoa through the human epididymis. Biol Reprod. 1988;39:812-7. PMID: 3207807 DOI: 10.1095/biolreprod39.4.812

Jurema MW, Vieira AD, Bankowski B, Petrella C, Zhao Y, Wallach E, Zacur H. Effect of ejaculatory abstinence period on the pregnancy rate after intrauterine insemination. Fertil Steril. 2005;84:678-81. PMID: 16169402 DOI: $10.1016 /$ j.fertnstert. 2005.03 .044

Lee J, Cha J, Shin S, Kim Y, Lee S, Cha H, Ahn J, Kim H, Pak K, Yoon J, Park S. Influence of abstinence period on clinical outcomes in fresh embryo transfer after intracytoplasmic sperm injection. Fertil Steril. 2015;104:E292. DOI: $10.1016 /$ j.fertnstert.2015.07.914

Lee JW, Cha JH, Shin SH, Cha HJ, Kim JH, Park CK, Pak KA, Yoon JS, Park SY. Effect of the sexual abstinence period recommended by the World Health Organization on clinical outcomes of fresh embryo transfer cycles with normal ovarian response after intracytoplasmic sperm injection. Andrologia. 2018;50:e12964. PMID: 29460293 DOI: 10.1111/and.12964

Levitas E, Lunenfeld E, Weiss N, Friger M, Har-Vardi I, Koifman A, Potashnik G. Relationship between the duration of sexual abstinence and semen quality: analysis of 9,489 semen samples. Fertil Steril. 2005;83:1680-6. PMID: 15950636 DOI: $10.1016 /$ j.fertnstert.2004.12.045
Marshburn PB, Alanis M, Matthews ML, Usadi R, Papadakis $\mathrm{MH}$, Kullstam S, Hurst BS. A short period of ejaculatory abstinence before intrauterine insemination is associated with higher pregnancy rates. Fertil Steril. 2010;93:286-8. PMID: 19732887 DOI: 10.1016/j.fertnstert.2009.07.972

Mayorga-Torres BJ, Camargo M, Agarwal A, du Plessis SS, Cadavid AP, Cardona Maya WD. Influence of ejaculation frequency on seminal parameters. Reprod Biol Endocrinol. 2015;13:47. PMID: 25994017 DOI: 10.1186/s12958-015-0045-9

Mazzilli R, Cimadomo D, Vaiarelli A, Capalbo A, Dovere L, Alviggi $E$, Dusi L, Foresta $C$, Lombardo F, Lenzi A, Tournaye $H$, Alviggi C, Rienzi L, Ubaldi FM. Effect of the male factor on the clinical outcome of intracytoplasmic sperm injection combined with preimplantation aneuploidy testing: observational longitudinal cohort study of 1,219 consecutive cycles. Fertil Steril. 2017;108:961-72.e3. PMID: 28985908 DOI: 10.1016/j. fertnstert.2017.08.033

Periyasamy AJ, Mahasampath G, Karthikeyan M, Mangalaraj AM, Kunjummen AT, Kamath MS. Does duration of abstinence affect the live-birth rate after assisted reproductive technology? A retrospective analysis of 1,030 cycles. Fertil Steril. 2017;108:988-92. PMID: 29100624 DOI: 10.1016/j.fertnstert.2017.08.034

Puissant F, Van Rysselberge M, Barlow P, Deweze J, Leroy F. Embryo scoring as a prognostic tool in IVF treatment. Hum Reprod. 1987;2:705-8. PMID: 3437050 DOI: 10.1093/oxfordjournals.humrep.a136618

Raziel A, Friedler S, Schachter M, Kaufman S, Omanski A, Soffer $Y$, Ron-El R. Influence of a short or long abstinence period on semen parameters in the ejaculate of patients with nonobstructive azoospermia. Fertil Steril. 2001;76:485-90. PMID: 11532469 DOI: 10.1016/S0015-0282(01)01956-2

Robaire B, Hinton BT, Orgebin-Crist MC. The epididymis. In Neill JD, ed. Knobil and Neill's Physiology of Reproduction. 3rd ed. San Diego: Academic Press/Elsevier; 2006. p. 1071-148.

Rowley MJ, Teshima F, Heller CG. Duration of transit of spermatozoa through the human male ductular system. Fertil Steril. 1970;21:390-6. PMID: 5508505 DOI: 10.1016/ S0015-0282(16)37502-1

Sánchez-Martín P, Sánchez-Martín F, González-Martínez M, Gosálvez J. Increased pregnancy after reduced male abstinence. Syst Biol Reprod Med. 2013;59:256-60. PMID: 23651301 DOI: 10.3109/19396368.2013.790919

Setti AS, Braga DPAF, Iaconelli Junior A, Borges Junior E. Increasing paternal age and ejaculatory abstinence length negatively influence the intracytoplasmic sperm injection outcomes from egg-sharing donation cycles. Andrology. 2020;8:594-601. PMID: 31755657 DOI: 10.1111/andr.12737

Sobreiro BP, Lucon AM, Pasqualotto FF, Hallak J, Athayde $\mathrm{KS}$, Arap S. Semen analysis in fertile patients undergoing vasectomy: reference values and variations according to age, length of sexual abstinence, seasonality, smoking habits and caffeine intake. Sao Paulo Med J. 2005;123:161-6. PMID: 16389413 DOI: 10.1590/S151631802005000400002 
Sugiyam R, Al-Salem JA, Nishi Y, Sugiyama R, Shirai A, Inoue $M$, Irahara $M$. Improvement of sperm motility by short-interval sequential ejaculation in oligoasthenozoospermic patients. Arch Med Sci. 2008;4:438-42.

Sunanda P, Panda B, Dash C, Padhy RN, Routray P. Effect of age and abstinence on semen quality: A retrospective study in a teaching hospital. Asian Pac J Reprod. 2014;3:134-41. DOI: 10.1016/S2305-0500(14)60017-8

Waller EJ, Roberts P, Matson P. Assessment of sperm motility, and its relationship with sperm function and fertility. J Reprod Biotechnol Fertil. 2019;8:30-53.
Welliver $C$, Benson AD, Frederick $L$, Leader B, Tirado E, Feustel P, Kontio J, McAsey M, Köhler TS. Analysis of semen parameters during 2 weeks of daily ejaculation: a first in humans study. Transl Androl Urol. 2016;5:749-55. PMID: 27785432 DOI: 10.21037/ tau.2016.08.20

WHO - World Health Organization. WHO laboratory manual for the examination and processing of human semen. 5th ed. Geneva: World Health Organization; 2010. 